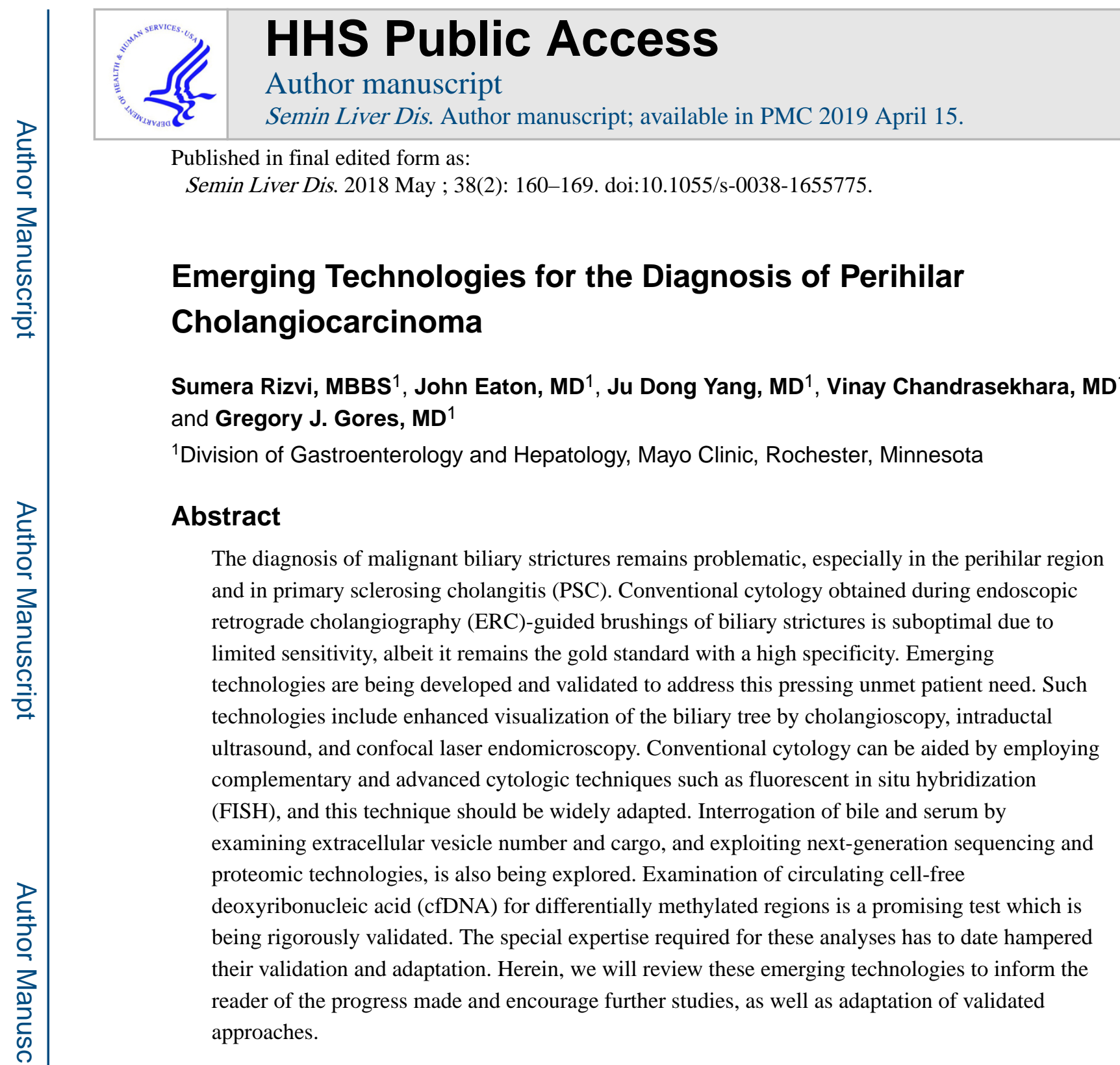

\title{
Keywords
}

cell-free DNA; differentially methylated regions; FISH

\begin{abstract}
Cholangiocarcinoma (CCA) represents a heterogeneous group of epithelial cell malignancies with features of cholangiocyte differentiation. ${ }^{1} \mathrm{CCA}$ is the most common biliary malignancy and the second most common hepatic malignancy (after hepatocellular carcinoma). CCAs are most commonly classified based on their anatomic location within the biliary tree into intrahepatic, perihilar (pCCA), and distal CCA (dCCA). pCCA involves the large bile ducts within the hepatic hilum and arises between the second-order biliary ducts proximally and the cystic duct insertion distally. ${ }^{2}$ pCCA is the most common subtype, representing $50 \%$ of CCA cases in a large series. ${ }^{3}$ Primary sclerosing cholangitis (PSC) is the most well-
\end{abstract}

\footnotetext{
Address for correspondence Gregory J. Gores, MD, Department of Medicine and Physiology, Mayo Clinic, 200 First Street SW, Rochester, MN 55905 (gores.gregory@ mayo.edu).
} 
established risk factor for pCCA. The 10-year cumulative incidence of CCA in PSC patients is 6 to $11 \%$ with a 30 -year risk of $20 \% .4,5$

CCAs are highly aggressive malignancies with a 5-year overall survival of less than $10 \%{ }^{6}$

Diagnosing pCCA at an early stage poses a significant challenge and contributes to the poor outcomes associated with this disease. Themajority of pCCA patients present with advanced-stage disease precluding curative treatment options such as surgical resection or neoadjuvant chemoradiation followed by liver transplantation. ${ }^{7}$ A significant factor in delayed diagnosis is that most patients do not become symptomatic until the cancer is advanced. Other diagnostic challenges include the remote anatomical location of pCCAs which often reside in difficult-to-access areas within the biliary tree, and the desmoplastic, paucicellular nature of these tumors. These factors limit the sensitivity of conventional cytology and biliary biopsy. For example, conventional cytology obtained by endoscopic retrograde cholangioscopy (ERC)-guided brushings only has a sensitivity of 20 to $40 \% .^{8,9}$ Moreover, the presence of inflammatory epithelial cell alterations in the setting of biliary infection or underlying PSC represents a diagnostic challenge on cytologic evaluation as these reactive cells often mimic cancer cells. ${ }^{10}$ Advanced cytological techniques ( $\square$ Fig. 1) such as fluorescence in situ hybridization (FISH) have improved sensitivity in combination with conventional biliary cytology. ${ }^{11}$ However, FISH analysis also has suboptimal sensitivity $(\sim 60 \%){ }^{8,12}$ Emerging techniques such as next-generation sequencing (NGS) and proteomic analysis have the potential to be viable adjunctive diagnostic tests for pCCA detection. Novel biomarkers such as circulating tumor cells (CTCs), circulating cell-free deoxyribonucleic acid (cfDNA), and extracellular vesicles (EVs) may overcome the sampling issues associated with direct biliary cytology and biopsy techniques. Herein, we review these novel biomarkers and advanced cytologic technologies in pCCA, along with advances in endoscopic techniques.

\section{Noninvasive Imaging}

Imaging is an indispensable tool for the detection of CCA. Ultrasonography, computed tomography (CT), positron emission tomography (PET), and magnetic resonance imaging(MRI)/magnetic resonance cholangiography (MRC) have been investigated as diagnostic imaging modalities.

In the absence of a mass, ultrasonography may only delineate intrahepatic ductal dilation, a common finding in PSC without a concomitant biliary cancer, without providing further details. ${ }^{13}$ Indeed, the sensitivity of ultrasound was only $10 \%$ among those with definitive evidence of CCA. Hence, it is an insufficient screening modality to detect early stage biliary cancers. ${ }^{14} \mathrm{CT}$ scans may detect mass lesions and investigate invasion into adjacent structures or metastases. The chief disadvantages of CT scans are the need for radiation as well as the limited ability to visualize the biliary tree and associated features which can be seen in early pCCA. PET scan rarely provides additive value when MRI/MRC and biliary brushings are inconclusive and false positive areas of 18-fluorodeoxyglucose (FDG) avidity due to inflammation are common. ${ }^{13,15}$ Recent data have suggested that standard uptake max may have prognostic implications and cut-offs may be able to distinguish between benign and malignant strictures among those with and without PSC. ${ }^{16,17}$ Larger cohorts comprised of 
PSC patients are needed to determine if these observations can be applied in this unique population. Among these modalities, MRI/MRC is the diagnostic technique of choice. Among patients with symptoms or laboratory tests suggestive of a biliary obstruction, MRI/MRC can provide useful information to the endoscopist prior to an ERC. The sensitivity and specificity of MRI/MRC to detect CCA is 88 to $89 \%$ and 75 to $85 \%$, respectively, and is superior to the sensitivity and specificity of CT (75-79\% and 79-80\%, respectively). ${ }^{14,18}$ A mass lesion with venous enhancement seen on MRI is very specific for CCA. ${ }^{19}$ However, a discrete mass is often absent in early stage disease. Imaging features that are indeterminate for CCA which should prompt an ERC include bile duct wall thickening, a bile duct stricture with or without proximal dilatation, duct wall irregularity, or contrast enhancement. ${ }^{20}$

\section{Advanced Endoscopic Techniques}

\section{Endoscopic Ultrasound}

Endoscopic ultrasound (EUS) allows for a detailed exam of the extrahepatic bile duct and surrounding structures from the duodenum, making it a valuable tool for the diagnosis and accurate staging of pCCA ( - Fig. 2). EUS is able to detect the primary tumor in $~ 94 \%$ of cases; however, in a large series, tumor detection was higher in dCCA (100\%) compared with proximal tumors (83\%). ${ }^{21}$ Whether or not to perform EUS-guided tissue acquisition of a pCCA has been debated in the literature. Sensitivity of EUS fine-needle aspiration (FNA) has been demonstrated to be lower for pCCA compared with dCCA (59\% vs. $81 \%) ;{ }^{21}$ however, a recent report suggests a higher diagnostic yield with EUS-guided tissue acquisition (82\%) in patients with suspected pCCA. ${ }^{22}$ A meta-analysis including 196 patients in 6 studies of EUS-FNA for the diagnosis of extrahepatic CCA demonstrated an overall pooled sensitivity of $66 \%$ (95\% confidence interval [CI]: $57-74 \%$ ). ${ }^{23}$ The benefits of EUS-FNA of primary tumors must be weighed against the risk of tumor dissemination. This risk was highlighted in a study of 191 patients with locally advanced CCA being evaluated for liver transplantation. ${ }^{24}$ Sixteen patients underwent transperitoneal FNA with peritoneal metastases identified in 5 of the 6 patients (83\%) with positive FNA cytology for malignancy compared with 14 of 175 who did not undergo transperitoneal FNA (8\%; $p=$ 0.0097). In a retrospective, single-center study of 150 consecutive patients with CCA, 61 patients underwent preoperative EUS-FNA of a primary biliary mass or stricture. ${ }^{25}$ In this study, performance of FNA did not adversely impact overall or progression-free survival; however, none of the patients underwent liver transplantation. The authors concluded that although tumor seeding is a theoretical risk with EUS-FNA, the clinical significance in patients with CCA is likely to be small. In that study, only $21 / 79$ (27\%) with pCCA underwent FNA compared with 40/53 (75\%) with dCCA. FNA of dCCA does not carry the same concern for tumor seeding as the duodenal wall is part of the resection field for curative-intent operations, whereas the duodenal bulb is not typically part of the resection field for pCCA. Hence, EUS-FNA of a pCCA is still believed to increase the risk of tumor dissemination and is considered an absolute contraindication to liver transplantation at the Mayo Clinic. ${ }^{26}$ 
Lymph node metastasis portends a poor outcome and therefore accurate staging is critical for individuals being considered for curative surgery. The role of EUS for nodal staging was investigated in a series of 47 patients with pCCA being considered for liver transplantation. EUS identified regional lymph nodes in all patients, of which 8 patients were confirmed to have malignant lymph nodes by FNA, whereas CT and/or MRI identified malignant lymphadenopathy in only 2 patients. ${ }^{27}$ Subsequent staging exploratory laparotomy confirmed the presence of benign lymph nodes in 20 of 22 patients with negative FNA (91\%). Morphological and echo features, including size, shape, echogenicity, and homogeneity, were of poor predictive value. Therefore, it is our practice to sample all visualized lymph nodes in patients with pCCA being considered for liver transplantation or curative resection. ${ }^{26}$

\section{ERC and Associated Technology}

ERC has played an important role for the anatomic delineation and tissue acquisition from suspected pCCA. ERC with brushing for cytology has been the standard approach for suspected malignant biliary strictures, but has been associated with low sensitivity when only positive for malignancy results (15\%) or when positive and suspicious for malignancy results $(38 \%)$ have been used. ${ }^{10}$ Various modifications of brush design and sampling technique have resulted in similarly suboptimal results. ERC with biliary biopsy has been associated with higher cancer detection rates, but a recent meta-analysis showed that this benefit was only demonstrated when biopsy was combined with brushing (59\% sensitivity) as the pooled sensitivity was similar between brushing (45\%) and intraductal biopsy (48\%). 28

\section{Intraductal Ultrasound}

Intraductal ultrasound (IDUS) is performed with a thin high-frequency probe that is advanced over a wire into the bile duct at the time of ERC. IDUS has been shown to improve diagnostic accuracy over traditional ERC-sampling techniques (e.g., conventional cytology has a sensitivity of 19-43\%). ${ }^{8}$ " In a study of 264 patients with biliary strictures that underwent surgical resection, IDUS was found to have a sensitivity, specificity, and accuracy of $93,89.5$, and $91 \%$, respectively. ${ }^{29}$ The main limitation of IDUS is that it is a visual diagnosis and lacks the ability to acquire tissue for confirmatory diagnosis. In addition, previously placed stents decrease the diagnostic yield of IDUS. ${ }^{30}$ IDUS provides limited nodal staging as the depth of radial penetration is $\sim 2 \mathrm{~cm}$. Although most endosonographers prefer conventional EUS as it provides superior locoregional staging and the ability to acquire tissue for cytology, IDUS may have a diagnostic role in patients with suspected pCCA not well visualized with prior imaging (including EUS) who do not have indwelling biliary stents.

\section{Cholangioscopy}

The development of single operator cholangioscopy (SOC) ( $\bullet$ Fig. 2) allowed for an easier platform for direct visualization of the biliary epithelium compared with percutaneous approaches and mother-daughter platforms that required two operators. In addition to providing visual information, SOC was believed to allow for targeted biopsies of biliary lesions thereby increasing the diagnostic yield of tissue sampling. In a large prospective 
multicenter study, Chen et al demonstrated that SOC visual impression had a sensitivity, specificity, positive predictive value, and negative predictive value of $78,82,80$, and $80 \%$ respectively. ${ }^{31}$ SOC-directed biopsies, however, demonstrated a lower overall sensitivity of $49 \%$ for malignancy, although this was higher for intrinsic bile duct malignancies (66\%). A recent systematic review of SOC-targeted biopsies for indeterminate biliary strictures identified 6 studies that reported an overall $66 \%$ sensitivity and $97 \%$ specificity to detect CCA. ${ }^{32}$ SOC with targeted biopsy sampling has been advocated as a cost-effective strategy for evaluating CCA in patients with PSC as compared with ERC with brushing for FISH with an incremental quality-adjusted life year gain of 0.22 at an additional cost of $\$ 8,562 .{ }^{33}$ Data using newer generation SOC systems with digital imaging are limited. A recent multicenter observational study that included 44 patients with indeterminate biliary strictures demonstrated a sensitivity and specificity of SOC digital visual impression for the diagnosis of malignancy of 90 and 96\%, respectively, and 85 and 100\%, respectively, for SOC-targeted biopsies. ${ }^{34}$ Similar digital imaging advancements have been achieved in choledochoscopes that are directly advanced into the bile duct after biliary sphincterotomy. An added benefit with these devices is that they allow for digital chromoendoscopy. In a study of 109 patients undergoing peroral video cholangiopancreatoscopy with narrow-band imaging that included 20 patients with biliary neoplasia, visual impression was associated with $85 \%$ sensitivity and $84 \%$ specificity. ${ }^{35}$ However, imaging directed sampling resulted in a lower sensitivity (43\%). Imaging features that were associated with neoplasia included tortuous and dilated vessels, infiltrative stricture, polypoid mass, and the presence of fish-egg lesions. While newer digital cholangioscopy platforms appear to have improved the ability to make a visual diagnosis of malignancy, it remains to be seen if these technologies can improve tissue diagnostic yield with targeted biopsy sampling.

\section{Confocal Laser Endomicroscopy}

Probe-based confocal laser endomicroscopy (CLE) allows for in vivo microscopic evaluation of the biliary epithelium, providing cellular and subcellular views by illuminating the tissue using a low-power laser and then detecting the reflected fluorescent light after administration of intravenous fluorescein. The CLE probe can be introduced through a catheter into the bile duct at the time of ERC. The probe must be in contact with the tissue for adequate imaging. In an effort to standardize imaging findings, the Miami classification system, which included the color and size of visualized bands as well as presence of dark clumps or epithelial structures, was proposed. ${ }^{36}$ Combining two or more criteria suggestive of malignancy provided a sensitivity, specificity, positive predictive value, and negative predictive value of $97,33,80$, and $80 \%$, respectively, compared with $48,100,100$, and $41 \%$ for standard tissue acquisition. ${ }^{36}$ Although the study authors reported moderate interobserver variability for most variables, subsequent studies demonstrated poor to fair interobserver agreement for individual criteria and poor to slight agreement for final interpretation of benign versus malignant lesions with CLE. ${ }^{37,38}$ The low specificity associated with the Miami classification was felt to be due to false-positive cases from benign inflammatory conditions. Consequently, the revised Paris criteria were developed that incorporated a third classification for findings associated with inflammatory biliary strictures. ${ }^{39}$ A prospective, international, multicenter study of 112 patients with indeterminate biliary strictures demonstrated that CLE had sensitivity, specificity, and accuracy of 89,71 , and $82 \%$, 
respectively, compared with 56,100 , and $72 \%$, respectively, for standard tissue sampling. ${ }^{40}$ Given the high level of expertise required to interpret CLE in real time along with concerns regarding standardizing training to improve interobserver agreement, it remains to be seen if these results can be replicated in other centers.

\section{Future Considerations}

Advanced endoscopic techniques are able to provide a more reliable visual assessment of the indeterminate biliary strictures; however, it remains to be seen if these technologies can aid in improved tissue acquisition for a diagnosis of pCCA. As current technologies applied in other areas of the digestive tract miniaturize, there may be an opportunity to better characterize biliary strictures with improved microscopic imaging platforms, computer-aided imaging analysis, and methods for assessing stiffness including EUS-based elastography or impedance planimetry.

\section{Advanced Cytologic Techniques}

\section{Biliary Cytology}

Biliary cytology is obtained by passing a wire brush across the biliary epithelium at the time of ERC or percutaneous cholangiography. Biliary biopsies can also be obtained and enhance the diagnostic yield. ${ }^{41}$ Biliary cytology can be classified into five categories: nondiagnostic, normal, atypical, suspicious, or positive for adenocarcinoma. ${ }^{42}$ Nondiagnostic cytology occurs when there is an insufficient amount of cellular material to review and should prompt resampling if there is a concern for CCA. Atypical cytology, which is often the product of biliary inflammation, is a common finding especially in PSC and by itself should not raise concern. ${ }^{43}$ In contrast, suspicious cytology is an independent predictor for CCA, and even in PSC nearly one-third of patients without a mass and suspicious cytology were eventually diagnosed with CCA. ${ }^{43,44}$ Cytology positive for adenocarcinoma is diagnostic of CCA. ${ }^{9,45}$ While the chief strength of cytology is its high specificity, the primary limitation is its limited sensitivity (19-43\%). ${ }^{8}, 9$ This is secondary to the desmoplastic, paucicellular nature of CCA which can be difficult to sample. Therefore, the absence of a positive cytology does not exclude malignancy.

\section{Fluorescence In Situ Hybridization}

FISH was developed as an objective test to identify aneuploidy, a marker of chromosomal instability. Approximately, $85 \%$ of biliary tract cancers display aneuploidy and, therefore, may be expected to be diagnosed by FISH. Because FISH samples a limited number of chromosomes, the term polysomy is used instead of aneuploidy. FISH polysomy in a de novo or sporadic perihilar stricture is virtually diagnostic of CCA ( $\downarrow$ Fig. 3). However, in PSC FISH may indicate dysplasia rather than invasive cancer. Hence, the role of FISH in PSC will be further discussed here.

The original UroVysion FISH assay utilizes three centromeric probes that target chromosomes 3,7, and 17 and a locus-specific probe to $9 \mathrm{p} 21$ from samples obtained by biliary brushings. FISH detects abnormal gains or losses of chromosomes and the results can be categorized as normal, trisomy (10 or more cells with 3 copies of chromosome 7 or 
chromosome 3, and 2 or fewer copies of the other 3 probes), tetrasomy (10 or more cells show 4 copies of all probes), or polysomy (5 or more cells show gains of 2 or more of the 4 probes). ${ }^{42}$

Polysomy (in contrast to a normal FISH result, trisomy or tetrasomy) is strongly associated with a diagnosis of CCA. ${ }^{43,44,46,47}$ A meta-analysis examined the performance of FISH testing among 690 PSC patients and the pooled sensitivity and specificity for polysomy and CCA was 51 and 93\%, respectively. ${ }^{12}$ A second-generation locus-specific FISH probe set (targets 1q21, 7p12,8q24, and 9p21) was recently derived and validated. ${ }^{8}$ Among those with PSC, the second-generation probe set had greater sensitivity than the UroVysion assay (65\% vs. $44 \%$ ) and a high specificity ( $90 \%)$ for the detection of CCA. ${ }^{8}$

FISH results should be interpreted in the context of each individual patient. The presence of other risk factors heightens the probability of harboring biliary cancer when polys-omy is detected. These additive risk factors include the presence of a "dominant stricture," elevated serum carbohydrate antigen 19-9 (CA 19-9), presence of suspicious cytology, multifocal, or serial polysomy ( $\triangle$ Table 1). ${ }^{43,44,46-48}$ The largest study $(n=371)$ that examined the natural history of FISH in PSC found that polysomy detected in multiple areas of the biliary tree (i.e., multifocal polysomy) was the strongest predictor of CCA. This study also noted that $71 \%$ of patients with CCA had polysomy detected at another region of the biliary tree where adenocarcinoma was not detected by routine cytology, which suggests that it would be helpful to sample multiple locations of the biliary tree. ${ }^{43}$

While the application of FISH to biliary brushings is a step forward, the test is hampered by limited sensitivity and suboptimal specificity (when compared with cytology) as not all patients with polysomy will develop CCA as they likely have nonprogressive low-grade dysplasia. Consequently, a new generation of biomarkers is needed to enhance clinicians' abilities to distinguish benign from malignant strictures in PSC.

\section{Application of Existing Technologies for Early CCA Detection and Screening}

Many experts in the field of PSC routinely screen their patients for CCA. ${ }^{20}$ There is some evidence to suggest that screening for CCA in PSC is associated with improved survival and detection at an earlier stage. ${ }^{49}$ The likelihood of CCA among asymptomatic pediatric patients or those with small duct PSC is low and they are not screened routinely. ${ }^{50,51} \mathrm{We}$ have previously published our approach to CCA screening and early detection in PSC. ${ }^{52}$ If there is a concern for underlying CCA after imaging, an ERC with biliary brushings for routine cytology/FISH studies and intraductal biopsies are recommended. Suspicious cytology is an independent predictor of CCA and needs follow-up. ${ }^{43,44,53}$ Indeed, an unpublished subgroup analysis from the aforementioned multifocal polysomy paper revealed that $14 \%$ (3/22) of patients with suspicious cytology alone (i.e., lack polysomy or a mass lesion) may have CCA diagnosed after a median follow-up of 1.5 years. ${ }^{43}$ The presence of polysomy should be confirmed on subsequent testing provided definitive evidence of CCA is absent. It may take more than 1 year for definitive evidence of CCA to manifest after polysomy is detected even among patients in an intensive surveillance program. $8,43,44,47$ Despite a lack of supporting or refuting evidence, we believe that this is a pragmatic 
approach with the potential to increase early detection and deepen the pool of subjects

eligible for curative therapy. ${ }^{42,52}$

\section{Evolving Techniques Including NGS, Extracellular Vesicles, and Proteomics NGS}

As conventional biliary cytology has limited sensitivity for the detection of pancreaticobiliary malignancy, adjunctive molecular testing such as FISH is necessary to improve on the sensitivity of biliary cytology. NGS is another adjunctive approach ( $\bullet$ Fig. 1) which has substantially improved the sensitivity of massive parallel sequencing by probing large panels of genes and identifying relatively rare mutations present in a small fraction of DNA templates. ${ }^{54}$ In an effort to compare the performance characteristics of NGS with FISH as adjunctive tests for the detection of pancreaticobiliary malignancy, bile duct ( $n=$ $73)$ and main pancreatic duct $(n=8)$ specimens from 74 patients who underwent endoscopic retrograde cholangiopancreatography (ERCP) were subjected to conventional cytology, FISH using the UroVysion probe set, and targeted NGS. ${ }^{55}$ On the basis of clinicopathologic follow-up, 33 specimens (41\%) were high-risk neoplasia/malignant strictures and 48 specimens (59\%) were benign. NGS combined with cytology had a sensitivity of $85 \%$ (compared with $67 \%$ for cytology alone), whereas FISH combined with cytology had a sensitivity of $76 \%$ for the detection of high-risk neoplasia or malignancy. ${ }^{55}$ NGS also revealed driver mutations in 24 cases (30\%). These findings indicate that NGS has the potential to be a viable adjunctive test for the detection of pancreaticobiliary malignancy. Moreover, NGS may have reduced cost and complexity compared with FISH as brushing samples subjected to NGS can be batched together with solid tumor specimens.

\section{EVs}

EVs are membrane-bound, heterogeneously sized vesicles released by diverse cell types which play an essential role in cell-to-cell communication. ${ }^{56} \mathrm{EVs}$ are released under both physiologic and pathologic conditions and carry cargo including proteins, lipids, and nucleic acids. Human biliary EVs also contain micro-ribonucleic acids (RNAs) (miRs) and long noncoding RNAs (lncRNAs). ${ }^{57,58} \mathrm{Li}$ et al identified a miR-based panel in biliary EVs with a sensitivity of $67 \%$ and specificity of $96 \% .{ }^{57}$ The same investigators identified EV-mediated trafficking of miR-195, downregulated in both cancer and stromal cells, between cancer cells and stromal cells. ${ }^{59}$ EVs containing lncRNAs have been implicated in tumor progression. ${ }^{58}$ Exosome sequencing analysis uncovered two lncRNAs with significantly increased expression in bile specimens of CCA patients compared with controls. ${ }^{58}$ The combination of these two IncRNAs had an area under the curve (AUC) of 0.709 in CCA detection. ${ }^{58}$ Tumorassociated microparticles (taMPs) are large EVs which may have a role in CCA detection. ${ }^{60}$ Annexin V + EpCAM + CD147+ taMPs enabled distinction between hepatic malignancy and cirrhosis in patients without malignancy ${ }^{60}$ However, it is unclear if Annexin V + EpCAM + CD147+ taMPs can distinguish between hepatocellular carcinoma and CCA as both malignancies were grouped together in this study. ${ }^{60} \mathrm{EVs}$ produced by cancer cells may carry oncogenic factors which promote tumorigenesis. ${ }^{61,62}$ A recent proteomic analysis demonstrated a higher abundance of oncogenic proteins in EVs obtained from human CCA cells compared with EVs obtained from normal human cholangiocytes. ${ }^{63}$ In this analysis, 
oncogenic proteins of particular interest included epidermal growth factor receptor, mucin-1, and integrin $\beta-4$ as these are upregulated in CCA and associated with CCA tumor growth and metastasis. ${ }^{63}$ Similar oncogenic proteins were noted in serum EVs of mice which had undergone orthotopic implantation of human CCA cells. ${ }^{63}$ Proteomic analysis using mass spectrometry demonstrated 95 differentially expressed proteins between healthy controls and CCA patients and 50 differentially expressed proteins between CCA patients versus PSC patients. ${ }^{63}$ From a functional standpoint, these proteins were primarily related to wound healing response, inflammatory response, and immune activation. ${ }^{63}$ Fibrinogen gamma chain, a-1-acid glycoprotein 1, and S100A8 were the three proteins with the best differential diagnosis capacity between CCA and PSC patients with AUC values of 0.796, 0.794, and 0.759 , respectively. ${ }^{63}$ The median concentration of EVs is also higher in bile specimens of patients with malignant biliary strictures compared with controls or nonmalignant biliary strictures $\left(2.4 \times 10^{15}\right.$ vs. $1.6 \times 10^{14}$ nanoparticles/L in the discovery cohort; $p<00.0001$, and $4.0 \times 10^{15}$ vs. $1.3 \times 10^{14}$ nanoparticles/L in the verification cohort; $\left.p<00.0001\right) .{ }^{64}$ Moreover, a bile EV threshold of $9.46 \times 10^{14}$ was able to distinguish malignant common bile duct stenoses from nonmalignant common bile duct stenoses with high diagnostic accuracy. 64

\section{Proteomics}

Other advanced technologies with the potential to enhance early CCA diagnosis include proteomic analysis by mass spectrometry or gel electrophoresis. Such analyses can detect novel biomarkers such as peptide panels in biological specimens including bile, serum, urine, and stool. For instance, a bile proteomic analysis by capillary electrophoresis mass spectrometry identified a 22-peptide panel in a training cohort of PSC $(n=18)$ and CCA ( $n$ $=16$ ) patients. ${ }^{65}$ In a subsequent validation cohort, this peptide panel accurately detected 14 of 18 bile specimens from PSC patients and 21 of 25 specimens from CCA patients (78\% specificity and $84 \%$ sensitivity). ${ }^{65}$ Similarly, a urine proteomic analysis using mass spectrometry identified a 42-peptide panel which differentiated CCA from PSC and benign biliary disorders with $83 \%$ sensitivity and $79 \%$ specificity. ${ }^{66}$ These data suggest a possible role for proteomic analysis in the detection of CCA in PSC patients, particularly when combined with noninvasive imaging studies such as MRI. However, prospective multicenter studies are necessary to validate these findings and to determine whether such peptide panels have utility in diagnosing CCA at an early stage.

\section{Evolving Techniques including Liquid Biopsy and CCA}

With recent advances in molecular technologies, genomics and epigenomic biomarkers display wide utility for early detection of various types of cancers. ${ }^{67} \mathrm{CTCs}$ and cfDNA ( Fig. 1) are novel biomarkers which may lead to improvements in patient outcomes by facilitating early detection of cancer. ${ }^{68}$ This new technology can reveal cancer-specific genetic and epigenetic changes directly from CTC and cfDNA in the blood stream. ${ }^{69}$ Circulating tumor biomarkers can overcome the sampling issues associated with cancer tissue biopsy as they reveal the whole spectrum of DNA mutations in cells released from heterogeneous cancer tissues, whereas tissue biopsy only reveals a snapshot of complex biology of tumors with extensive intra- and interlesional diversity. ${ }^{70,71}$ Genomic and 
epigenomic analyses from the peripheral blood will therefore help to develop a noninvasive accurate diagnostic approach in the era of individualized medicine. While CTC are typically detected in patients with advanced stage cancer, a role for CTC in screening and early detection of cancer was reported in other cancers such as lung, breast, and pancreatic cancer. ${ }^{72-74}$ Detection of CTC was associated with a 10-fold increased risk of having shorter survival among patients with pCCA/dCCA ${ }^{75}$ Diagnostic role of CTC in early detection of CCA remains to be elucidated and a prospective study is ongoing to address this question.

An accumulating body of literature confirms an excellent correlation between DNA mutations found in cancer tissues and mutations detected in cfDNA from the same patients. In one study of 17 patients with pancreatobiliary malignancy reported by Zill et al, $90.3 \%$ of mutations detected in tumor biopsies were also detected in cfDNA. ${ }^{76}$ While excellent concordances in tissue and cfDNA mutations were reported, all patients had advanced stage disease. Hence, the sensitivity of cfDNA mutations for early stage tumor detection is currently unknown. The sensitivity of detection of methylated DNA changes in cfDNA has improved; consequently, differentially methylated regions (DMRs) in cfDNA are often measurable in patients without detectable CTC and have the potential to be an excellent diagnostic biomarker for CCA. Andresen et al reported a four-gene DNA methylation biomarker panel from biliary brush samples, which achieved a sensitivity of $85 \%$ and a specificity of $98 \%$ for CCA detection, with an AUC of $0.944 .{ }^{77}$ A similar pilot study using cfDNA methylation as a diagnostic biomarker identified the top nine DNA methylation makers using tissue methylome analysis. ${ }^{78}$ These methylation markers were tested on cfDNA obtained from $2 \mathrm{~mL}$ of plasma of CCA patients ( $N=69: 48$ de novo, 21 PSCassociated) and age-sex matched healthy controls $(N=95) .{ }^{78}$ The recursive partitioning decision tree method identified a 4-DMR panel, which classified CCA with a sensitivity of $83 \%$ and a specificity of $93 \%$ with AUC of $0.90 .{ }^{78}$ Model calls were not significantly influenced by comorbid PSC or tumor stage. A prospective study is ongoing to confirm the diagnostic performance of cfDNA methylation marker in a larger number of samples.

\section{Conclusion and Future Directions}

To date, conventional cytology via ERC-guided acquisition approaches is most widely practiced for the diagnosis of malignant strictures. However, it is imperative that this approach be supplanted by improved techniques. Too often, decision making is deferred because the cytology is non-diagnostic. The diagnosis of pCCA will always require a cellular and/or genetic indicator of malignancy along with imaging evidence compatible with a cancer. Therefore, surrogate endpoints such as EVs without genetic cargo analysis, proteomics, and/or imaging technologies in isolation are unlikely to be diagnostic. It is also unlikely that further advances in cytologic techniques (e.g., FISH, machine learning employing conventional cytologic specimens, etc.) will be sufficiently more diagnostic than current assessments. This relates, in part, to the paucicellular nature of the specimens, and the highly desmoplastic nature of these cancers. Furthermore, sample acquisition following placement of biliary stents also confounds these diagnostic approaches. Likely, easy-toemploy biliary imaging techniques along with cfDNA technology from the bile and/or blood will improve our ability to diagnose this enigmatic cancer. We encourage clinical 
investigators in partnership with diagnostic pathologists to develop protocols along these lines to address this unmet clinical need.

\section{Acknowledgments}

This work was supported by National Institutes of Health grant DK59427 (G.J.G.), and the Mayo Foundation. S. Rizvi also received support from the Cholangiocarcinoma Foundation, the Satter Foundation Liver Cancer Award, and a Pilot \& Feasibility Award by the Center for Cell Signaling in Gastroenterology (P30DK084567). We thank Ms. Courtney Hoover for excellent secretarial support.

\section{References}

1. Rizvi S, Gores GJ. Pathogenesis, diagnosis, and management of cholangiocarcinoma. Gastroenterology 2013;145(06):1215-1229 [PubMed: 24140396]

2. Rizvi S, Gores GJ. Current diagnostic and management options in perihilar cholangiocarcinoma. Digestion 2014;89(03):216-224 [PubMed: 24860985]

3. DeOliveira ML, Cunningham SC, Cameron JL, et al. Cholangiocarcinoma: thirty-one-year experience with 564 patients at a single institution. Ann Surg 2007;245(05):755-762 [PubMed: 17457168]

4. Boonstra K, Weersma RK, van Erpecum KJ, et al.; EpiPSCPBC Study Group. Population-based epidemiology, malignancy risk, and outcome of primary sclerosing cholangitis. Hepatology 2013;58(06):2045-2055 [PubMed: 23775876]

5. Kornfeld D, Ekbom A, Ihre T. Survival and risk of cholangiocarcinoma in patients with primary sclerosing cholangitis. A population-based study. Scand J Gastroenterol 1997;32(10):1042-1045 [PubMed: 9361178]

6. Everhart JE, Ruhl CE. Burden of digestive diseases in the United States Part III: liver, biliary tract, and pancreas. Gastroenterology 2009;136(04):1134-1144 [PubMed: 19245868]

7. Rizvi S, Khan SA, Hallemeier CL, Kelley RK, Gores GJ. Cholangio-carcinoma - evolving concepts and therapeutic strategies. Nat Rev Clin Oncol 2018;15(02):95-111 [PubMed: 28994423]

8. Barr Fritcher EG, Voss JS, Brankley SM, et al. An optimized set of fluorescence in situ hybridization probes for detection of pancreatobiliary tract cancer in cytology brush samples. Gastroenterology 2015;149(07):1813-1824.e1 [PubMed: 26327129]

9. Trikudanathan G, Navaneethan U, Njei B, Vargo JJ, Parsi MA. Diagnostic yield of bile duct brushings for cholangiocarcinoma in primary sclerosing cholangitis: a systematic review and metaanalysis. Gastrointest Endosc 2014;79(05):783-789 [PubMed: 24140129]

10. Barr Fritcher EG, Kipp BR, Slezak JM, et al. Correlating routine cytology, quantitative nuclear morphometry by digital image analysis, and genetic alterations by fluorescence in situ hybridization to assess the sensitivity of cytology for detecting pancreatobiliary tract malignancy. Am J Clin Pathol 2007;128(02):272-279 [PubMed: 17638662]

11. Brooks C, Gausman V, Kokoy-Mondragon C, et al. Role of fluorescent in situ hybridization, cholangioscopic biopsies, and EUS-FNA in the evaluation of biliary strictures. Dig Dis Sci 2018;63(03):636-644 [PubMed: 29353443]

12. Navaneethan U, Njei B, Venkatesh PG, Vargo JJ, Parsi MA. Fluorescence in situ hybridization for diagnosis of cholangiocarcinoma in primary sclerosing cholangitis: a systematic review and metaanalysis. Gastrointest Endosc 2014;79(06):943-950.e3 [PubMed: 24360654]

13. Jhaveri KS, Hosseini-Nik H. MRI of cholangiocarcinoma. J Magn Reson Imaging 2015;42(05): 1165-1179 [PubMed: 25447417]

14. Charatcharoenwitthaya P, Enders FB, Halling KC, Lindor KD. Utility of serum tumor markers, imaging, and biliary cytology for detecting cholangiocarcinoma in primary sclerosing cholangitis. Hepatology 2008;48(04):1106-1117 [PubMed: 18785620]

15. Fevery J, Buchel O, Nevens F, Verslype C, Stroobants S, Van Steenbergen W. Positron emission tomography is not a reliable method for the early diagnosis ofcholangiocarcinoma in patients with primary sclerosing cholangitis. J Hepatol 2005;43(02):358-360 [PubMed: 15975687] 
16. Alkhawaldeh K, Faltten S, Biersack HJ, Ezziddin S. The value of F-18 FDG PET in patients with primary sclerosing cholangitis and cholangiocarcinoma using visual and semiquantitative analysis. Clin Nucl Med 2011;36(10):879-883 [PubMed: 21892037]

17. Ma KW, Cheung TT, She WH, et al. Diagnostic and prognostic role of 18-FDG PET/CT in the management of resectable biliary tract cancer. World J Surg 2018;42(03):823-834 [PubMed: 28905105]

18. Saluja SS, Sharma R, Pal S, Sahni P, Chattopadhyay TK. Differentiation between benign and malignant hilar obstructions using laboratory and radiological investigations: a prospective study. HPB 2007;9(05):373-382 [PubMed: 18345322]

19. Chapman R, Fevery J, Kalloo A, et al.; American Association for the Study of Liver Diseases. Diagnosis and management of primary sclerosing cholangitis. Hepatology 2010;51(02):660-678 [PubMed: 20101749]

20. Schramm C, Eaton J, Ringe KI, Venkatesh S, Yamamura J; MRI working group of the IPSCSG. Recommendations on the use of magnetic resonance imaging in PSC-A position statement from the International PSC Study Group. Hepatology 2017;66(05):1675-1688 [PubMed: 28555945]

21. Mohamadnejad M, DeWitt JM, Sherman S, et al. Role of EUS for preoperative evaluation of cholangiocarcinoma: a large single-center experience. Gastrointest Endosc 2011;73(01):71-78 [PubMed: 21067747]

22. Téllez-Ávila FI, Bernal-Méndez AR, Guerrero-Vázquez CG, Martínez-Lozano JA, Ramírez-Luna MÁ. Diagnostic yield of EUS-guided tissue acquisition as a first-line approach in patients with suspected hilar cholangiocarcinoma. Am J Gastroenterol 2014;109(08):1294-1296 [PubMed: 25091254]

23. Navaneethan U, Njei B, Venkatesh PG, Lourdusamy V, Sanaka MR. Endoscopic ultrasound in the diagnosis of cholangiocarcinoma as the etiology of biliary strictures: a systematic review and meta-analysis. Gastroenterol Rep (Oxf) 2015;3(03):209-215 [PubMed: 25169922]

24. Heimbach JK, Sanchez W, Rosen CB, Gores GJ. Trans-peritoneal fine needle aspiration biopsy of hilar cholangiocarcinoma is associated with disease dissemination. HPB 2011;13(05):356-360 [PubMed: 21492336]

25. El Chafic AH, Dewitt J, Leblanc JK, et al. Impact of preoperative endoscopic ultrasound-guided fine needle aspiration on post-operative recurrence and survival in cholangiocarcinoma patients. Endoscopy 2013;45(11):883-889 [PubMed: 24165813]

26. Levy MJ, Heimbach JK, Gores GJ. Endoscopic ultrasound staging of cholangiocarcinoma. Curr Opin Gastroenterol 2012;28(03):244-252 [PubMed: 22274618]

27. Gleeson FC, Rajan E, Levy MJ, et al. EUS-guided FNA of regional lymph nodes in patients with unresectable hilar cholangiocarcinoma. Gastrointest Endosc 2008;67(03):438-443 [PubMed: 18061597]

28. Navaneethan U, Njei B, Lourdusamy V, Konjeti R, Vargo JJ, Parsi MA. Comparative effectiveness of biliary brush cytology and intraductal biopsy for detection of malignant biliary strictures: a systematic review and meta-analysis. Gastrointest Endosc 2015;81(01):168-176 [PubMed: 25440678]

29. Meister T, Heinzow HS, Woestmeyer C, et al. Intraductal ultra-sound substantiates diagnostics of bile duct strictures of uncertain etiology. World J Gastroenterol 2013;19(06):874-881 [PubMed: 23430958]

30. Lee JH, Salem R, Aslanian H, Chacho M, Topazian M. Endoscopic ultrasound and fine-needle aspiration of unexplained bile duct strictures. Am J Gastroenterol 2004;99(06):1069-1073 [PubMed: 15180727]

31. Chen YK, Parsi MA, Binmoeller KF, et al. Single-operator cholangioscopy in patients requiring evaluation of bile duct disease or therapy of biliary stones (with videos). Gastrointest Endosc 2011;74(04):805-814 [PubMed: 21762903]

32. Navaneethan U, Hasan MK, Lourdusamy V, Njei B, Varadarajulu S, Hawes RH. Single-operator cholangioscopy and targeted biopsies in the diagnosis of indeterminate biliary strictures: a systematic review. Gastrointest Endosc 2015;82(04):608-14.e2 [PubMed: 26071061] 
33. Njei B, McCarty TR, Varadarajulu S, Navaneethan U. Cost utility of ERCP-based modalities for the diagnosis of cholangiocarcinoma in primary sclerosing cholangitis. Gastrointest Endosc 2017;85(04):773-781.e10 [PubMed: 27590963]

34. Navaneethan U, Hasan MK, Kommaraju K, et al. Digital, single-operator cholangiopancreatoscopy in the diagnosis and management of pancreatobiliary disorders: a multicenter clinical experience (with video). Gastrointest Endosc 2016;84(04):649-655 [PubMed: 26995690]

35. Mounzer R, Austin GL, Wani S, Brauer BC, Fukami N, Shah RJ. Peroral video cholangiopancreatoscopy with narrow-band imaging for the evaluation of indeterminate pancreaticobiliary disease. Gastrointest Endosc 2017;85(03):509-517 [PubMed: 27894928]

36. Meining A, Shah RJ, Slivka A, et al. Classification of probe-based confocal laser endomicroscopy findings in pancreaticobiliary strictures. Endoscopy 2012;44(03):251-257 [PubMed: 22261749]

37. Talreja JP, Sethi A, Jamidar PA, et al. Interpretation of probe-based confocal laser endomicroscopy of indeterminate biliary strictures: is there any interobserver agreement? Dig Dis Sci 2012;57(12): 3299-3302 [PubMed: 22875310]

38. Peter S, Council L, Bang JY, et al. Poor agreement between endoscopists and gastrointestinal pathologists for the interpretation of probe-based confocal laser endomicroscopy findings. World J Gastroenterol 2014;20(47):17993-18000 [PubMed: 25548499]

39. Caillol F, Filoche B, Gaidhane M, Kahaleh M. Refined probe-based confocal laser endomicroscopy classification for biliary strictures: the Paris Classification. Dig Dis Sci 2013;58(06):1784-1789 [PubMed: 23314855]

40. Slivka A, Gan I, Jamidar P, et al. Validation of the diagnostic accuracy of probe-based confocal laser endomicroscopy for the characterization of indeterminate biliary strictures: results of a prospective multicenter international study. Gastrointest Endosc 2015;81(02):282-290 [PubMed: 25616752]

41. Jailwala J, Fogel EL, Sherman S, et al. Triple-tissue sampling at ERCP in malignant biliary obstruction. Gastrointest Endosc 2000;51(4 Pt 1):383-390 [PubMed: 10744806]

42. Eaton JE, Gossard AA, Talwalkar JA. Recall processes for biliary cytology in primary sclerosing cholangitis. Curr Opin Gastroenterol 2014;30(03):287-294 [PubMed: 24686433]

43. Eaton JE, Barr Fritcher EG, Gores GJ, et al. Biliary multifocal chromosomal polysomy and cholangiocarcinoma in primary sclerosing cholangitis. Am J Gastroenterol 2015;110(02):299-309 [PubMed: 25623660]

44. Barr Fritcher EG, Voss JS, Jenkins SM, et al. Primary sclerosing cholangitis with equivocal cytology: fluorescence in situ hybridization and serum CA 19-9 predict risk of malignancy. Cancer Cytopathol 2013;121(12):708-717 [PubMed: 23839915]

45. Furmanczyk PS, Grieco VS, Agoff SN. Biliary brush cytology and the detection of cholangiocarcinoma in primary sclerosing cholangitis: evaluation of specific cytomorphologic features and CA19-9 levels. Am J Clin Pathol 2005;124(03):355-360 [PubMed: 16191503]

46. Bangarulingam SY, Bjornsson E, Enders F, et al. Long-term outcomes of positive fluorescence in situ hybridization tests in primary sclerosing cholangitis. Hepatology 2010;51(01):174-180 [PubMed: 19877179]

47. Barr Fritcher EG, Kipp BR, Voss JS, et al. Primary sclerosing cholangitis patients with serial polysomy fluorescence in situ hybridization results are at increased risk of cholangiocarcinoma. Am J Gastroenterol 2011;106(11):2023-2028 [PubMed: 21844920]

48. Levy C, Lymp J, Angulo P, Gores GJ, Larusso N, Lindor KD. The value of serum CA 19-9 in predicting cholangiocarcinomas in patients with primary sclerosing cholangitis. Dig Dis Sci 2005;50(09):1734-1740 [PubMed: 16133981]

49. Ali AH, Tabibian JH, Nasser-Ghodsi N, et al. Surveillance for hepatobiliary cancers in patients with primary sclerosing cholangitis. Hepatology 2017 Doi: 10.1002/hep.29730

50. Eaton JE, McCauley BM, Atkinson EJ, et al. Variations in primary sclerosing cholangitis across the age spectrum. J Gastroenterol Hepatol 2017;32(10):1763-1768 [PubMed: 28245345]

51. Weismüller TJ, Trivedi PJ, Bergquist A, et al.; International PSC Study Group. Patient age, sex, and inflammatory bowel disease phenotype associate with course of primary sclerosing cholangitis. Gastroenterology 2017;152(08):1975-1984.e8 [PubMed: 28274849] 
52. Rizvi S, Eaton JE, Gores GJ. Primary sclerosing cholangitis as a premalignant biliary tract disease: surveillance and management. Clin Gastroenterol Hepatol 2015;13(12):2152-2165 [PubMed: 26051390]

53. Boyd S, Tenca A, Jokelainen K, et al. Screening primary sclerosing cholangitis and biliary dysplasia with endoscopic retrograde cholangiography and brush cytology: risk factors for biliary neoplasia. Endoscopy 2016;48(05):432-439 [PubMed: 26808393]

54. Kinde I, Wu J, Papadopoulos N, Kinzler KW, Vogelstein B. Detection and quantification of rare mutations with massively parallel sequencing. Proc Natl Acad Sci U S A 2011;108(23):9530-9535 [PubMed: 21586637]

55. Dudley JC, Zheng Z, McDonald T, et al. Next-generation sequencing and fluorescence in situ hybridization have comparable performance characteristics in the analysis of pancreaticobiliary brushings for malignancy. J Mol Diagn 2016;18(01):124-130 [PubMed: 26596524]

56. Hirsova P, Ibrahim SH, Verma VK, et al. Extracellular vesicles in liver pathobiology: small particles with big impact. Hepatology 2016;64(06):2219-2233 [PubMed: 27628960]

57. Li L, Masica D, Ishida M, et al. Human bile contains microRNA-laden extracellular vesicles that can be used for cholangiocarci-noma diagnosis. Hepatology 2014;60(03):896-907 [PubMed: 24497320]

58. Ge X, Wang Y, Nie J, et al. The diagnostic/prognostic potential and molecular functions of long non-coding RNAs in the exosomes derived from the bile of human cholangiocarcinoma. Oncotarget 2017;8(41):69995-70005 [PubMed: 29050258]

59. Li L, Piontek K, Ishida M, et al. Extracellular vesicles carry micro-RNA-195 to intrahepatic cholangiocarcinoma and improve survival in a rat model. Hepatology 2017;65(02):501-514 [PubMed: 27474881]

60. Julich-Haertel H, Urban SK, Krawczyk M, et al. Cancer-associated circulating large extracellular vesicles in cholangiocarcinoma and hepatocellular carcinoma. J Hepatol 2017;67(02):282-292 [PubMed: 28267620]

61. Al-Nedawi K, Meehan B, Micallef J, et al. Intercellular transfer of the oncogenic receptor EGFRvIII by microvesicles derived from tumour cells. Nat Cell Biol 2008;10(05):619-624 [PubMed: 18425114]

62. Peinado H, Aleçković M, Lavotshkin S, et al. Melanoma exosomes educate bone marrow progenitor cells toward a pro-metastatic phenotype through MET. Nat Med 2012;18(06):883-891 [PubMed: 22635005]

63. Arbelaiz A, Azkargorta M, Krawczyk M, et al. Serum extracellular vesicles contain protein biomarkers for primary sclerosing cholangitis and cholangiocarcinoma. Hepatology 2017;66(04): 1125-1143 [PubMed: 28555885]

64. Severino V, Dumonceau JM, Delhaye M, et al. Extracellular vesicles in bile as markers of malignant biliary stenoses. Gastroenterology 2017;153(02):495-504.e8 [PubMed: 28479376]

65. Lankisch TO, Metzger J, Negm AA, et al. Bile proteomic profiles differentiate cholangiocarcinoma from primary sclerosing cholangitis and choledocholithiasis. Hepatology 2011;53(03):875-884 [PubMed: 21374660]

66. Metzger J, Negm AA, Plentz RR, et al. Urine proteomic analysis differentiates cholangiocarcinoma from primary sclerosing cholangitis and other benign biliary disorders. Gut 2013;62(01):122-130 [PubMed: 22580416]

67. Alix-Panabières C, Pantel K. Clinical applications of circulating tumor cells and circulating tumor DNA as liquid biopsy. Cancer Discov 2016;6(05):479-491 [PubMed: 26969689]

68. Haber DA, Velculescu VE. Blood-based analyses of cancer: circulating tumor cells and circulating tumor DNA. Cancer Discov 2014;4(06):650-661 [PubMed: 24801577]

69. Pantel K, Alix-Panabières C. Real-time liquid biopsy in cancer patients: fact or fiction? Cancer Res 2013;73(21):6384-6388 [PubMed: 24145355]

70. Gerlinger M, Rowan AJ, Horswell S, et al. Intratumor heterogeneity and branched evolution revealed by multiregion sequencing. N Engl J Med 2012;366(10):883-892 [PubMed: 22397650]

71. Murtaza M, Dawson SJ, Pogrebniak K, et al. Multifocal clonal evolution characterized using circulating tumour DNA in a case of metastatic breast cancer. Nat Commun 2015;6:8760 [PubMed: 26530965] 
72. Ilie M, Hofman V, Long-Mira E, et al. "Sentinel" circulating tumor cells allow early diagnosis of lung cancer in patients with chronic obstructive pulmonary disease. PLoS One 2014;9(10):e111597 [PubMed: 25360587]

73. Rhim AD, Mirek ET, Aiello NM, et al. EMT and dissemination precede pancreatic tumor formation. Cell 2012;148(1-2):349-361 [PubMed: 22265420]

74. Hüsemann Y, Geigl JB, Schubert F, et al. Systemic spread is an early step in breast cancer. Cancer Cell 2008;13(01):58-68 [PubMed: 18167340]

75. Yang JD, Campion MB, Liu MC, et al. Circulating tumor cells are associated with poor overall survival in patients with cholangio-carcinoma. Hepatology 2016;63(01):148-158 [PubMed: 26096702]

76. Zill OA, Greene C, Sebisanovic D, et al. Cell-free DNA next-generation sequencing in pancreatobiliary carcinomas. Cancer Discov 2015;5(10):1040-1048 [PubMed: 26109333]

77. Andresen K, Boberg KM, Vedeld HM, et al. Four DNA methylation biomarkers in biliary brush samples accurately identify the presence of cholangiocarcinoma. Hepatology 2015;61(05):16511659 [PubMed: 25644509]

78. Yang J, Yab T, Taylor WR, et al. Detection of cholangiocarcinoma by assay of methylated DNA markers in plasma. Gastroenterology 2017;152:S1041-S1042 


\section{Peripheral circulation}

- Tumor markers (e.g. CA 19-9)

- Circulating tumor cells

- Differentially methylated regions in cell-free DNA

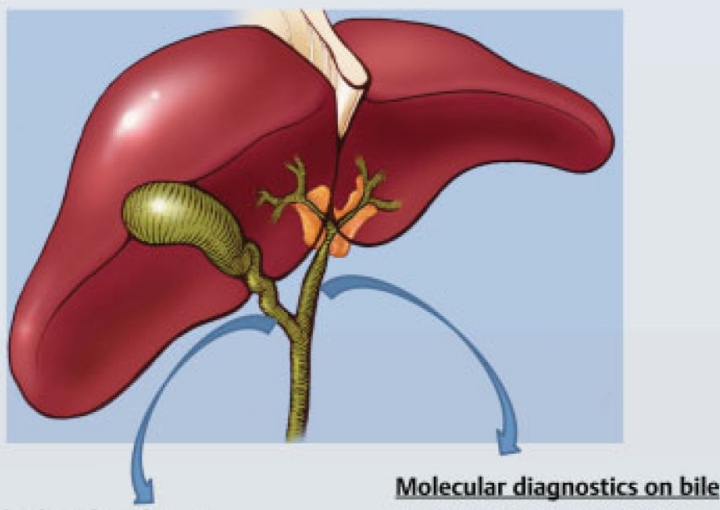

Assays on bile duct brushings

- Conventional cytology

Molecular diagnostics on bile

- Fluorescence in situ hybridization

- Next-generation sequencing

- MicroRNAs in extracellular vesicles

- Proteomics

- Mutational profiling of cell-free DNA

\section{Fig. 1.}

Diagnostic modalities in perihilar cholangiocarcinoma (pCCA). Conventional and emerging diagnostic techniques performed on serum, bile duct brushings, and bile specimens for the detection of pCCA. 


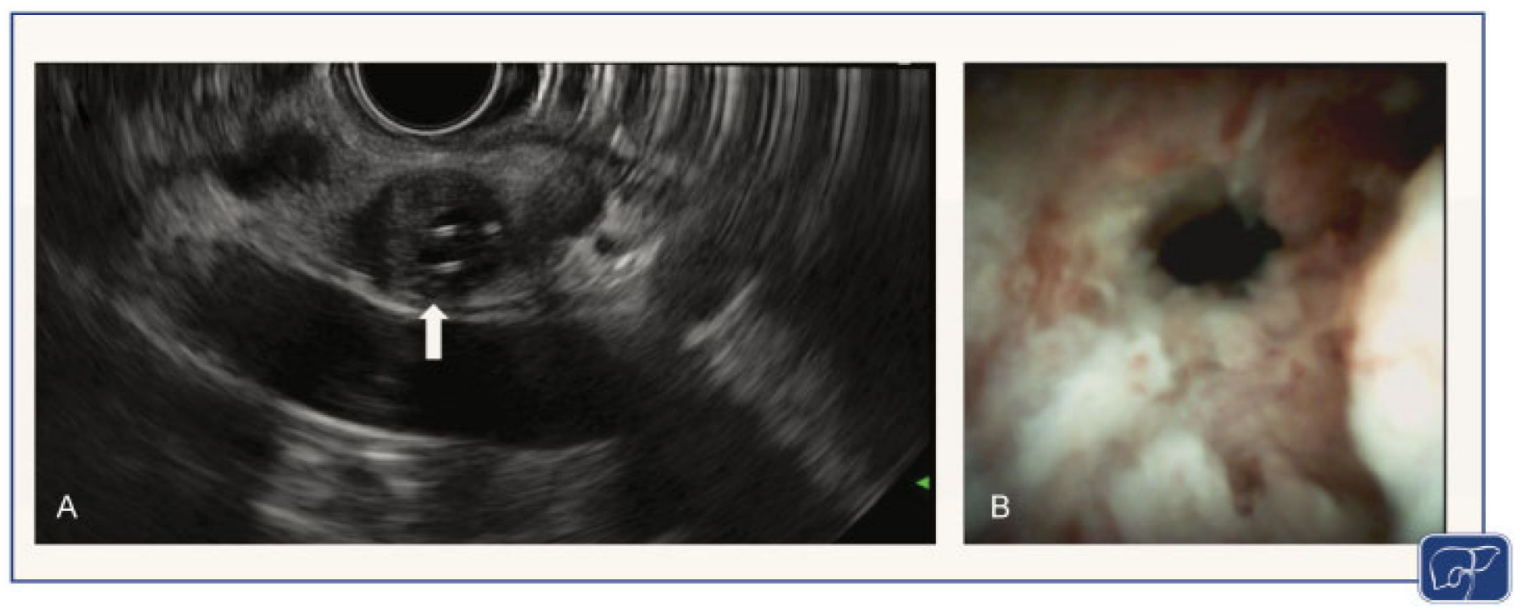

Fig. 2.

Advanced endoscopic techniques for the diagnosis of perihilar cholangiocarcinoma (pCCA). (A) Digital cholangioscopy demonstrating an infiltrative stricture with dilated vessels in a patient without a mass seen on cross-sectional imaging. Cholangioscopy-targeted biopsies confirmed the presence of adenocarcinoma. (B) Endoscopic ultrasound (EUS) image demonstrating a 1-cm mass (white arrow in $\mathbf{A}$ ) of the common hepatic duct, corresponding with a perihilar cholangiocarcinoma with a plastic stent traversing within the bile duct. 


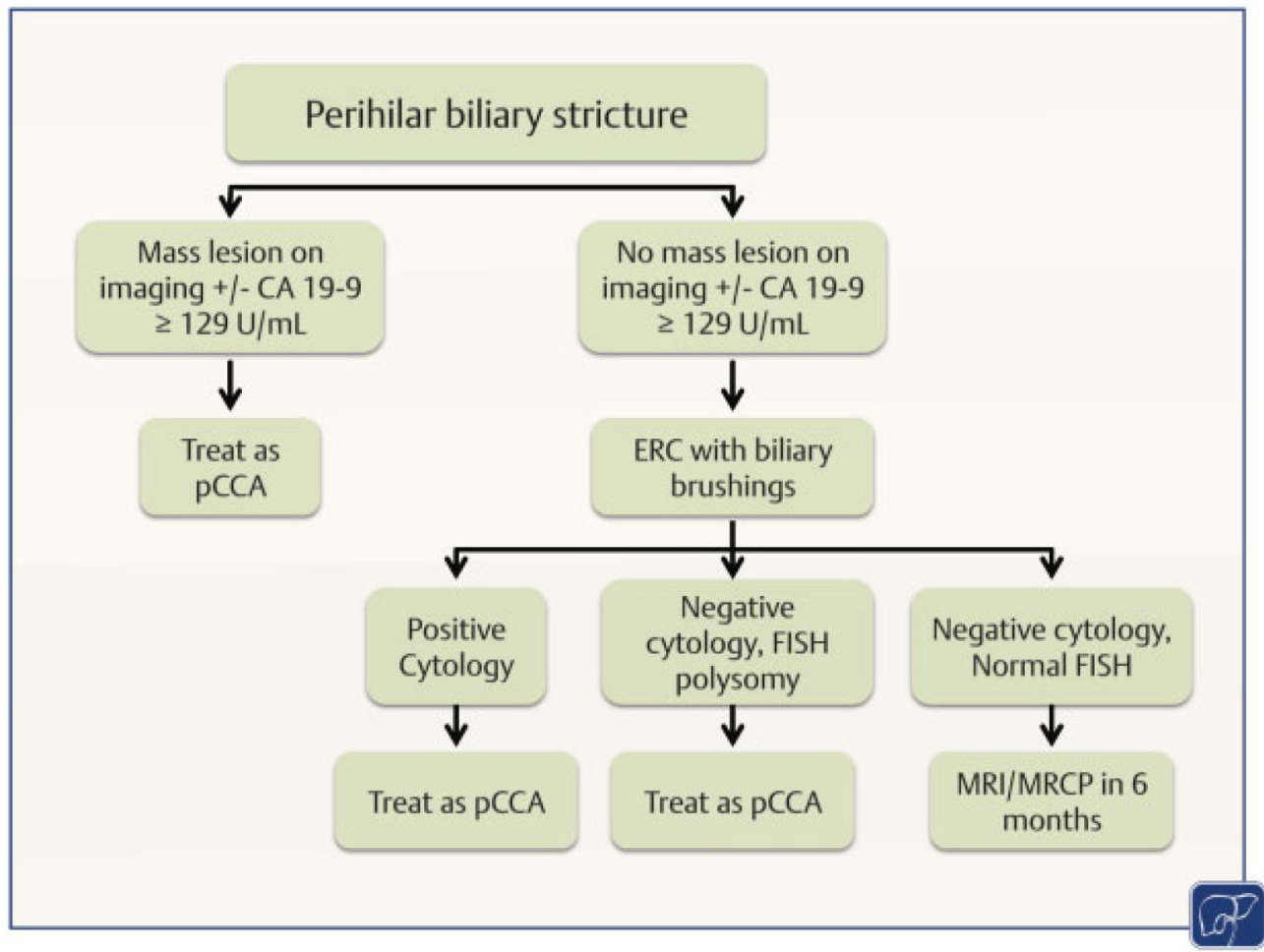

Fig. 3.

Approach to a sporadic (de novo) perihilar biliary stricture. Management algorithm for patients with a sporadic or de novo perihilar biliary stricture. 
